\title{
Three-Dimensional Visualization of Nichols, Hall, and Robust-Performance Diagrams
}

\author{
Kent H. Lundberg and Zachary J. Malchano \\ Department of Electrical Engineering and Computer Science \\ Massachusetts Institute of Technology, Cambridge, MA 02139
}

\begin{abstract}
Three-dimensional models and animations of Nichols charts, Hall charts, and robust-performance diagrams are presented. Using these models, students can visualize the implications and importance of these charts and diagrams. By viewing these animations, students develop better intuition concerning the connection between open-loop gain/phase plots, open-loop polar plots, and closed-loop frequency response.
\end{abstract}

\section{INTRODUCTION}

Upon first presentation, the Nichols chart and the Hall chart are often confusing to first-term control students. This paper describes several educational animations that help students visualize the implications and importance of Nichols charts and Hall charts by showing the magnitude of the closed-loop frequency response as the third dimension.

Robust performance, in the face of multiplicative plant uncertainty, can be illustrated with two disjoint sets of frequencydependent circles on a Nyquist diagram (one set for plant uncertainty and the other set for specifications on sensitivity). However, distinct circles must be plotted at all frequencies. By using the third dimension for frequency, a Nyquist plot can be rendered that includes the effect of plant uncertainty at all frequencies. Examining this model for intersections of the corresponding solids indicates the success or failure of a robust-performance design.

\section{NichOLS CHART}

The Nichols chart [1] has been used for many years to bridge the gap between open-loop frequency response and closed-loop frequency response. Plotting the open-loop frequency response on gain/phase coordinates allows the closedloop frequency response to be read from the chart. Unfortunately, students are often bewildered by the complicated chart when it is first presented, likening it to a plate of spaghetti or a sadistic dart board, as shown in Figure 1.

A change in point of view can help students appreciate the importance of the chart. The Nichols chart can be thought of as a contour map of Mount Nichols (Figure 2) where the height of the mountain corresponds to the magnitude of the closedloop frequency response for all possible values of open-loop frequency response. When the frequency response of a specific loop transfer function for a unity-feedback system, such as

$$
L_{1}(s)=\frac{1}{s(s+1)},
$$

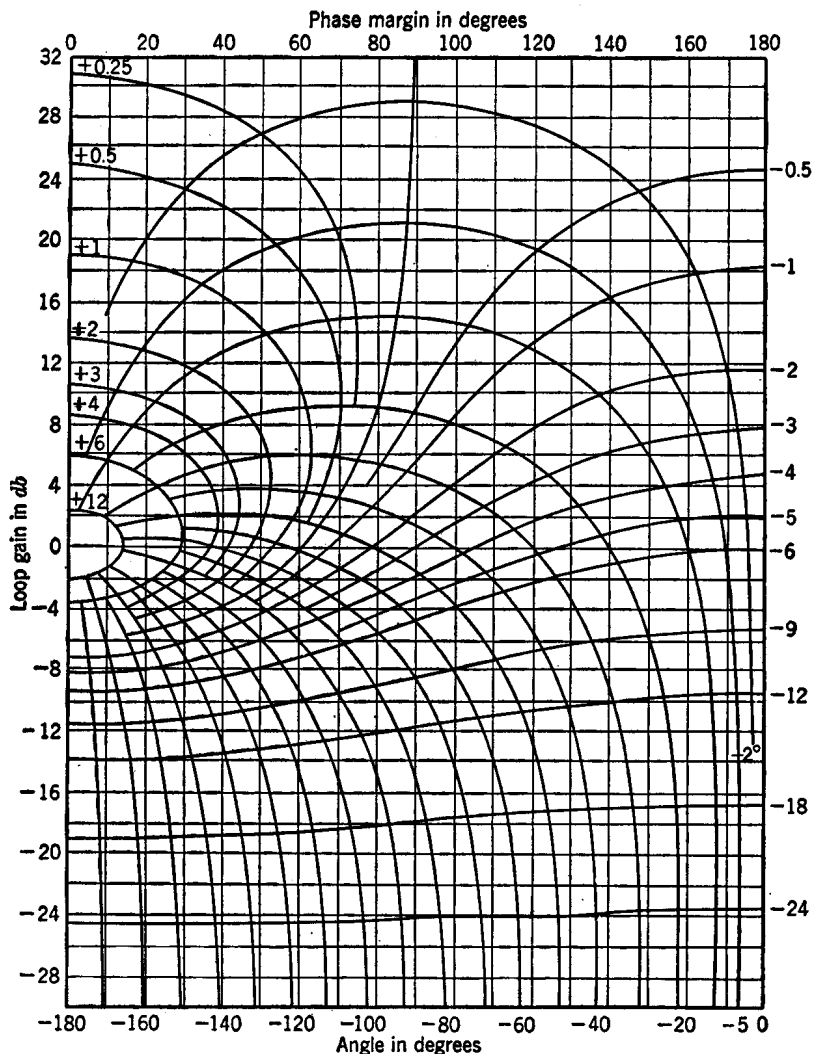

Fig. 1. The original Nichols chart, reprinted from the 1947 textbook by James, Nichols, and Phillips [1]. The curves show contours of constant closedloop magnitude and constant closed-loop phase. To use the chart, the openloop frequency response is plotted on the rectilinear gain/phase coordinates, and the closed-loop frequency response is read from the contours of closedloop magnitude and phase.

is plotted on the gain/phase plane as shown in Figure 3, the height of the surface of Mount Nichols corresponds to the magnitude of the closed-loop frequency response for that $L(j \omega)$ as shown in Figure 4.

Showing Mount Nichols and its infinite peak also reinforces the danger of getting too close to the $s=-1$ point, as illustrated by

$$
L_{2}(s)=\frac{10}{s(s+1)}
$$

shown by the upper curves in Figures 5 and 6. As the openloop frequency response gets closer the the peak of Mount 


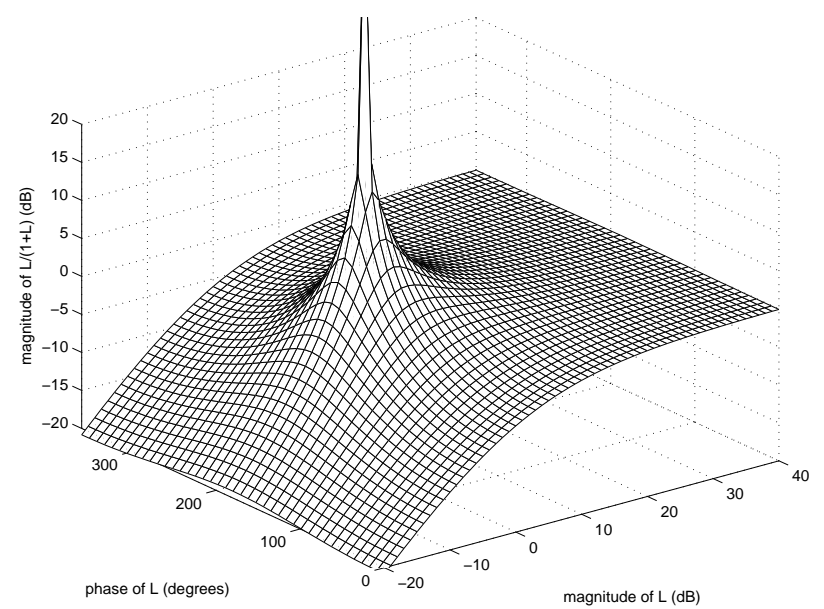

Fig. 2. Mount Nichols. The height of the mountain corresponds to the magnitude of the closed-loop frequency response. When the open-loop frequency response is plotted on the rectilinear gain/phase coordinates on the horizontal plane, the magnitude of the closed-loop frequency response is shown by the height of the surface.

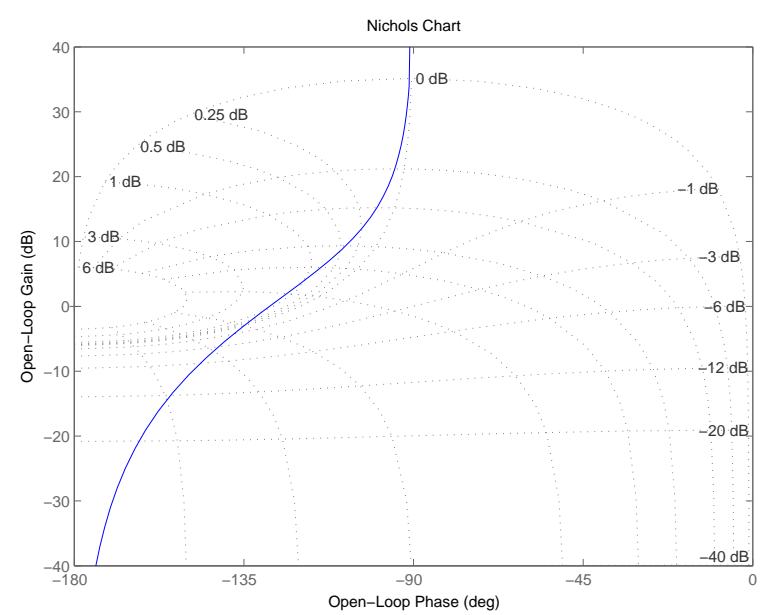

Fig. 3. Nichols chart for $L_{1}(s)$. The closed-loop frequency response is read from the contours of closed-loop magnitude.

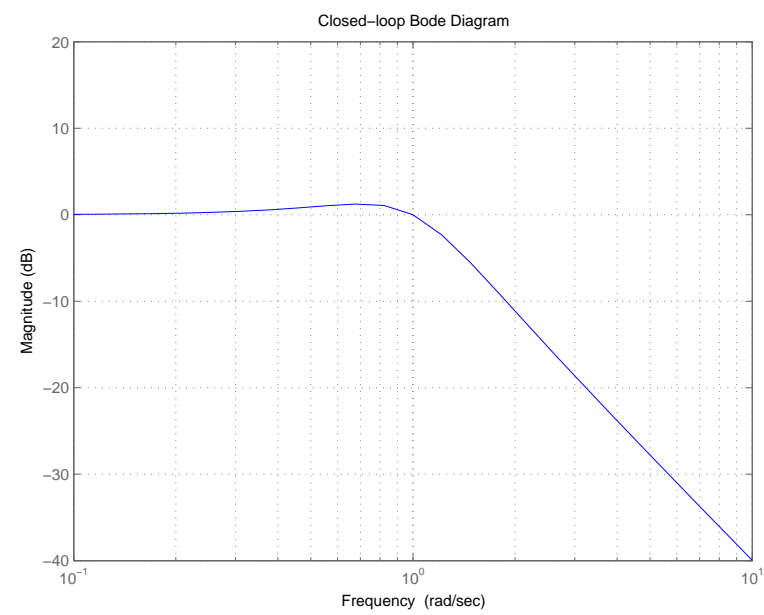

Fig. 4. Closed-loop Bode plot for $L_{1}(s)$. The slight peaking in the response agrees with the 1-dB contour in Figure 3.

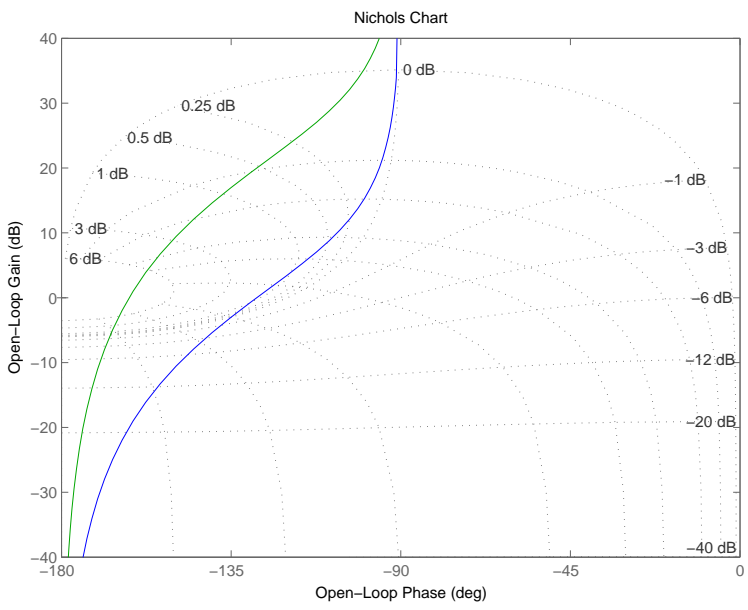

Fig. 5. Nichols chart for $L_{1}(s)$ and $L_{2}(s)$. The change in gain between $L_{1}(s)$ and $L_{2}(s)$ corresponds to a shift up the vertical axis. Since $L_{2}(j \omega)$ approaches closer to the $s=-1$ point, the closed-loop frequency response will appear much higher up the "foothills" of Mount Nichols.

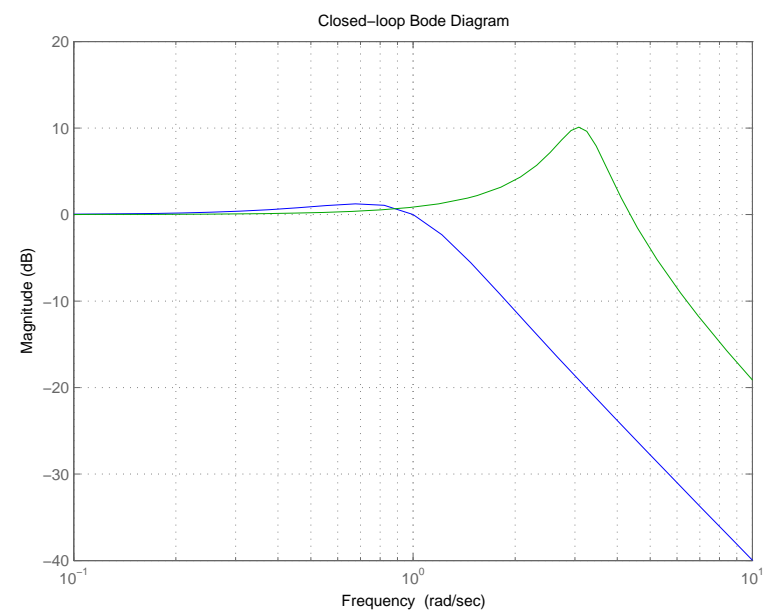

Fig. 6. Closed-loop Bode plot for $L_{1}(s)$ and $L_{2}(s)$. The extreme increase in the magnitude peaking for $L_{2}(s)$ is due to its height on Mount Nichols. This correlation between height on Mount Nichols and closed-loop magnitude peaking is demonstrated by the animations on the next page.

Nichols, the peaking in the closed-loop frequency response gets larger.

This transformation from the open-loop frequency response to the closed-loop frequency response is illustrated by a computer animation that starts with the gain/phase plot on the surface of Mount Nichols as viewed from above. The camera view is rotated to show the elevation of the curve on Mount Nichols to demonstrate that the height of the open-loop frequency response corresponds to the closed-loop frequency response.

The animation can be repeated for multiple loop transfer functions, such as (1) and (2). Still frames from these animations are shown in Figures 7 and 8, respectively. 

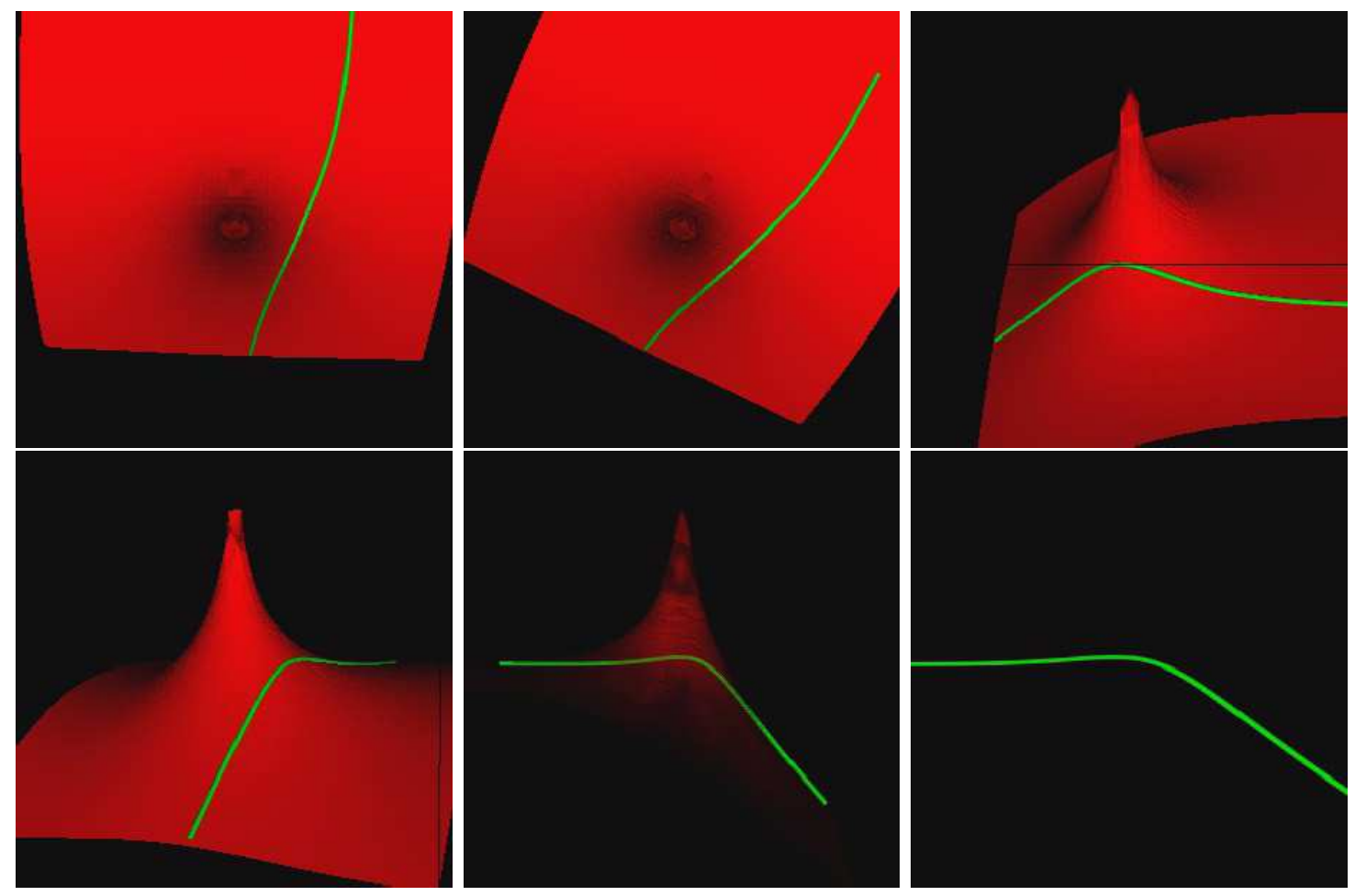

Fig. 7. Still images from Nichols animation for $L_{1}(s)=\frac{1}{s(s+1)}$.
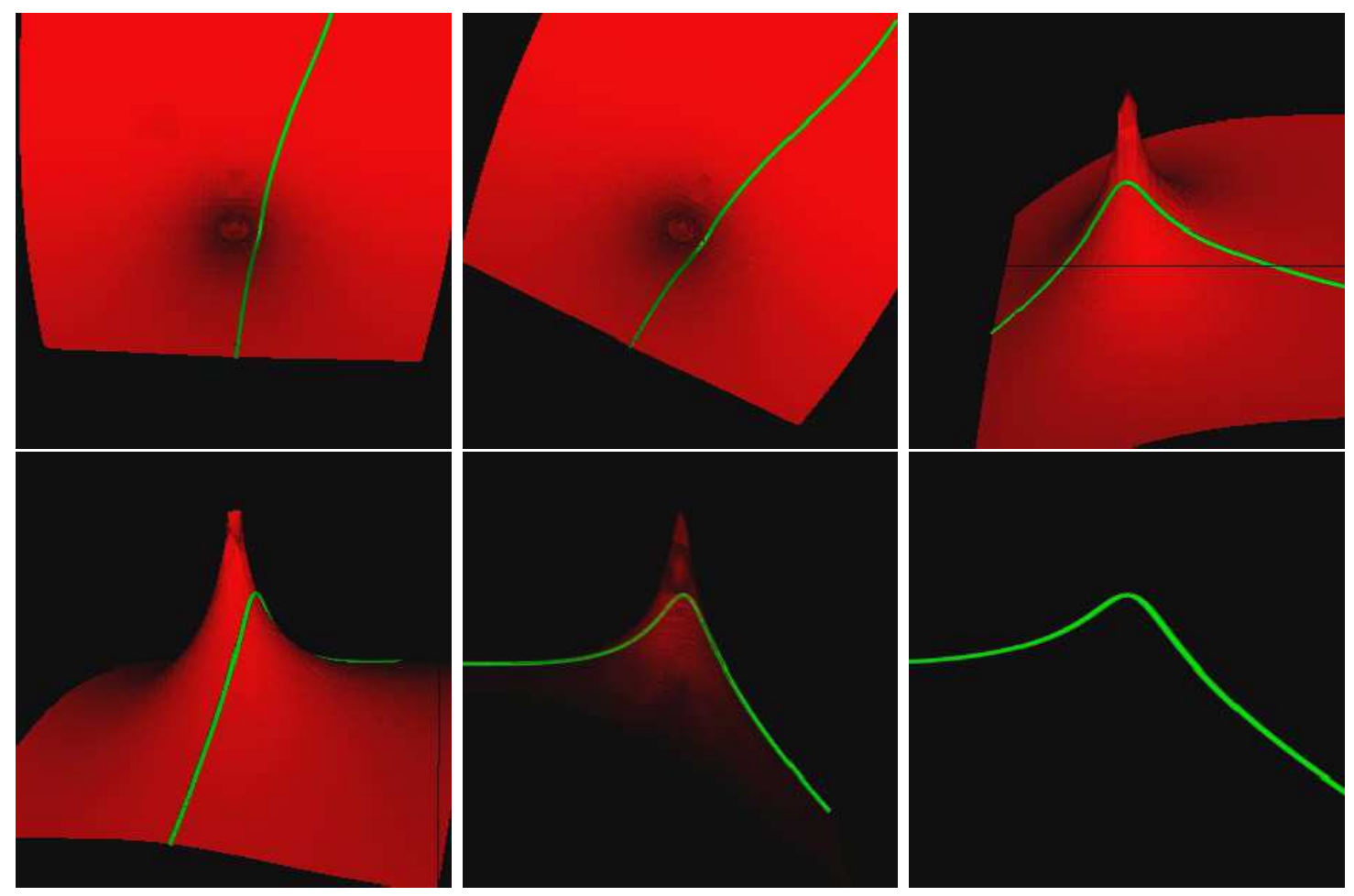

Fig. 8. Still images from Nichols animation for $L_{2}(s)=\frac{10}{s(s+1)}$. 


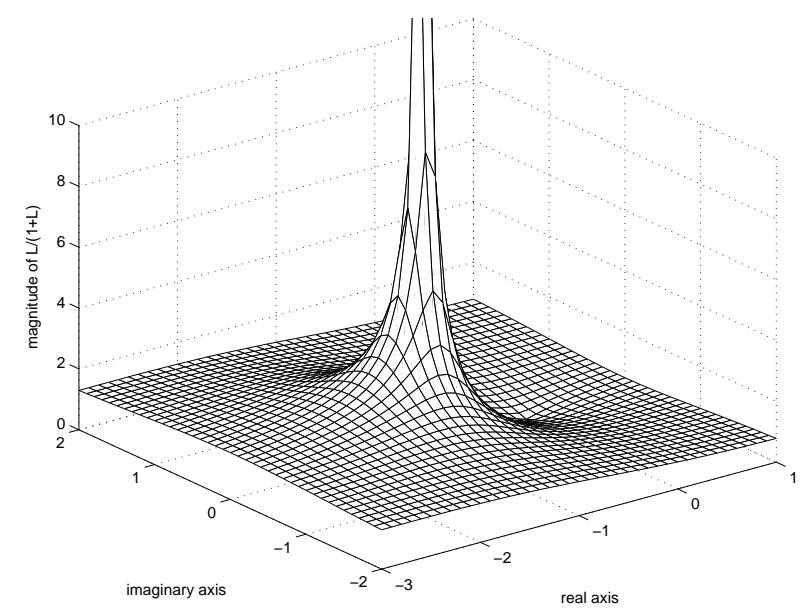

Fig. 9. Mount Hall. The height of the mountain corresponds to the magnitude of the closed-loop frequency response. When the open-loop frequency response is plotted on the Nyquist coordinates on the horizontal plane, the magnitude of the closed-loop frequency response is shown by the height of the surface.

\section{HALL CHART}

The Hall chart [2] predates the Nichols chart by several years, but is not as popular with control educators. Pedagogically, the Hall chart is a powerful tool, since it reinforces the relationship between the Nyquist plot and the Bode plot. In addition, the $M$-circles of the Hall chart can be used in robust Nyquist diagrams, and early introduction of these concepts can improve student understanding and intuition.

A similar change in perspective can help students appreciate the importance of the Hall chart. The Hall chart is a contour map of Mount Hall (Figure 9) where the height of the mountain corresponds to the magnitude of the closed-loop frequency response for all possible values of the open-loop frequency response. Mount Hall has an infinite peak at the $s=-1$ point, and it has a dimple to zero at the origin, as we expect from the magnitude of the closed-loop transfer function

$$
M=\left|\frac{L}{1+L}\right| .
$$

Figures 10 and 11 show the loop transfer functions (1) and (2) plotted with the Hall chart. Reading the $M$-circles on the Hall chart provides the magnitude of the closed-loop frequency response, as shown in Figure 12.

Animations similar to those shown in Figures 7 and 8 are made to help students visualize the connection between the polar plot of the open-loop frequency response and the Bode plot of the closed-loop frequency response. The animation starts with the polar plot on the surface of Mount Hall as viewed from above. The camera view is rotated to show the elevation of the curve on Mount Hall to demonstrate that the height of the open-loop frequency response corresponds to the closed-loop frequency response.

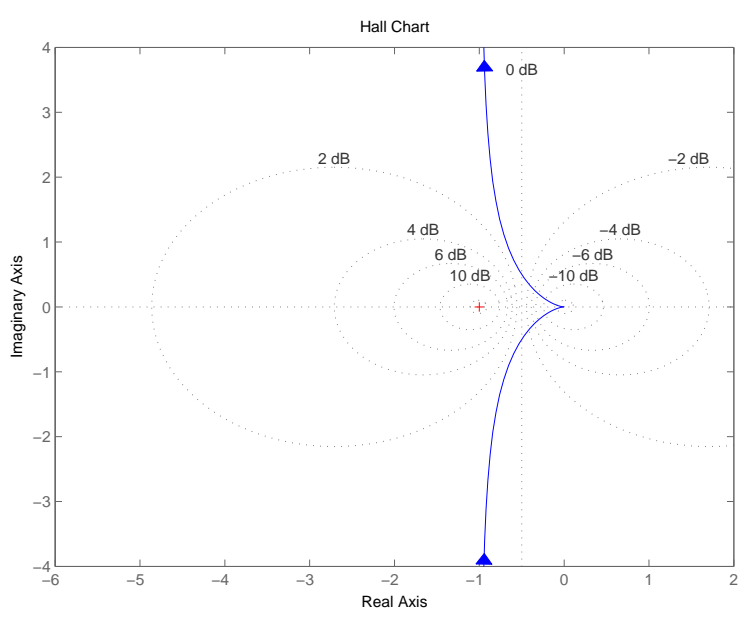

Fig. 10. Hall chart for $L_{1}(s)$. The closed-loop frequency response is read from the contours of closed-loop magnitude.

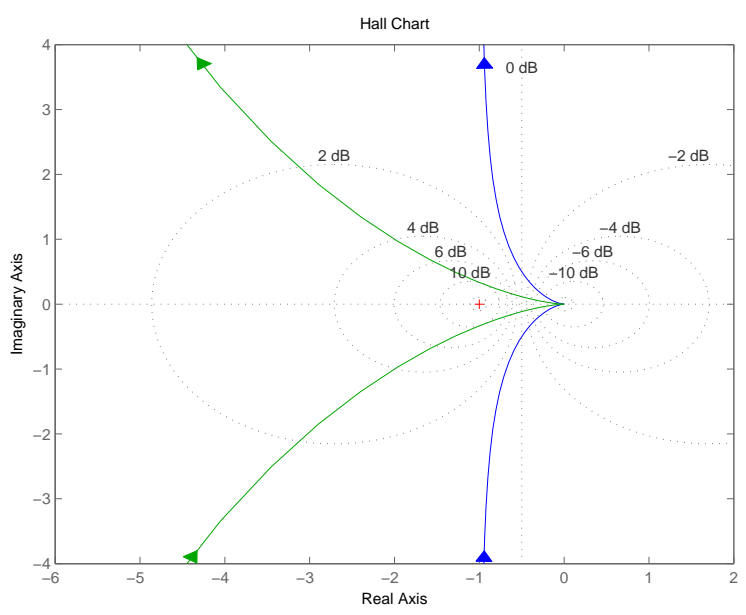

Fig. 11. Hall chart for $L_{1}(s)$ and $L_{2}(s)$. The frequency response $L_{2}(j \omega)$ is closer to the $s=-1$ point and the peak of Mount Hall.

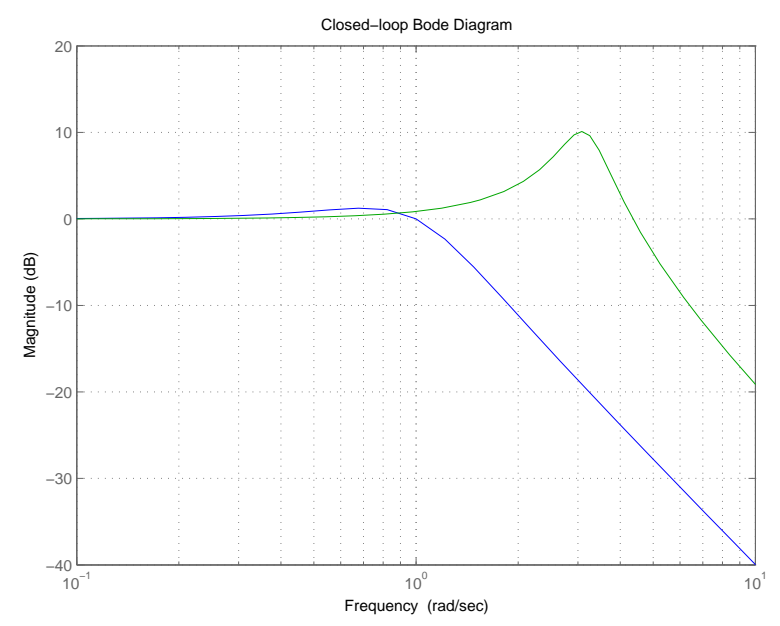

Fig. 12. Closed-loop Bode plot for $L_{1}(s)$ and $L_{2}(s)$. The 10-dB peaking in the response agrees with the $10-\mathrm{dB}$ contour in Figure 11 and the associated height on the foothills of Mount Hall. 


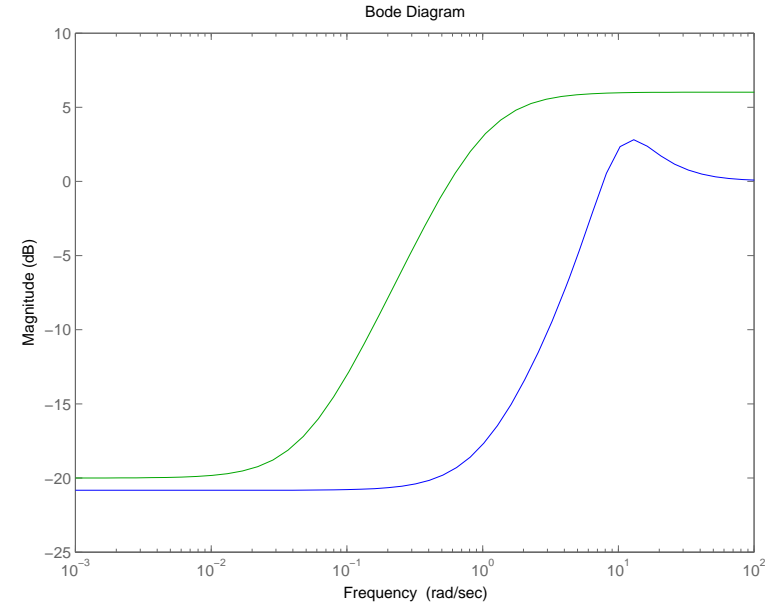

Fig. 13. Magnitude plot of nominal-plant sensitivity transfer function and sensitivity bound $W_{1}(j \omega)$. For the system to meet the specified requirements on dynamic-tracking error, the sensitivity transfer function must always be below the bound $W_{1}(j \omega)$.

\section{Robust-Performance Diagrams}

Three-dimensional visualizations can also help students understand the frequency dependence of robust-performance diagrams. A robust-performance diagram shows the competing bounds on sensitivity and uncertainty, which must not intersect for a successfully robust design. However, the usual way of plotting these diagrams [3] can be confusing.

As an example, consider a nominal-plant transfer function

$$
L(s)=\frac{10}{(s+1)(0.1 s+1)}
$$

The requirements for a small dynamic-tracking error can be specified in terms of a sensitivity bound

$$
\left|\frac{1}{1+L(j \omega)}\right| \leq \frac{1}{\left|W_{1}(j \omega)\right|}
$$

For a particular set of dynamic-tracking requirements, the sensitivity bound could be expressed as

$$
W_{1}^{-1}(s)=\frac{0.1(20 s+1)}{s+1}
$$

as shown in the Bode plot in Figure 13.

The sensitivity bound can also be written in terms of a vector length from the $s=-1$ point [3] as

$$
|1+L(j \omega)|>\left|W_{1}(j \omega)\right|
$$

Therefore, the sensitivity bound can also be displayed on a Nyquist plot, as shown in Figure 14. At each frequency $\omega_{k}$, the loop transfer function $L\left(j \omega_{k}\right)$ must be outside the circle of radius $\left|W_{1}\left(j \omega_{k}\right)\right|$ centered at $s=-1$.

Now consider a perturbed plant transfer function with an unmodeled high-frequency pole

$$
L^{\prime}(s)=\frac{10}{(s+1)(0.1 s+1)}\left(\frac{1}{\tau s+1}\right)
$$

where it is known that $\tau<\tau_{m}=0.04$ second.

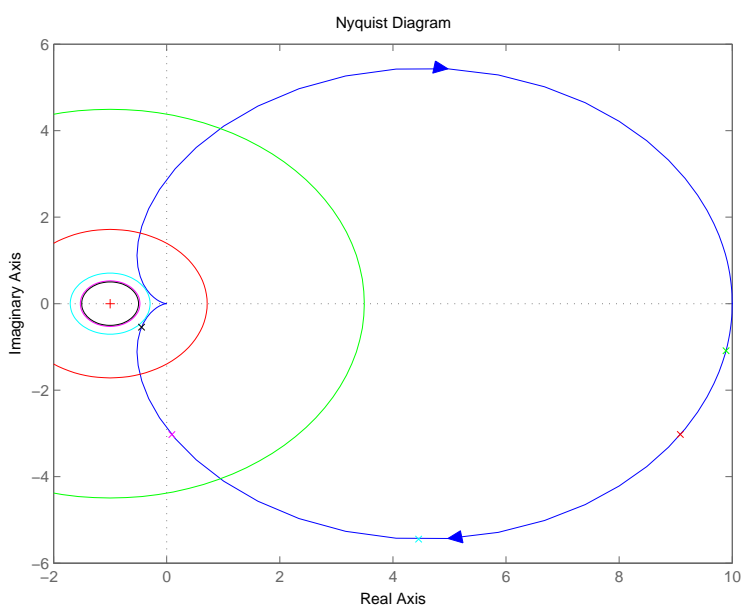

Fig. 14. Performance chart showing nominal-plant loop transfer function $L(j \omega)$ and frequency-dependent sensitivity bound $W_{1}(j \omega)$. At each frequency, the loop transfer function $L$ (shown by each colored"x") must be outside the circle of radius $\left|W_{1}\right|$ (of the same color) centered at $s=-1$.

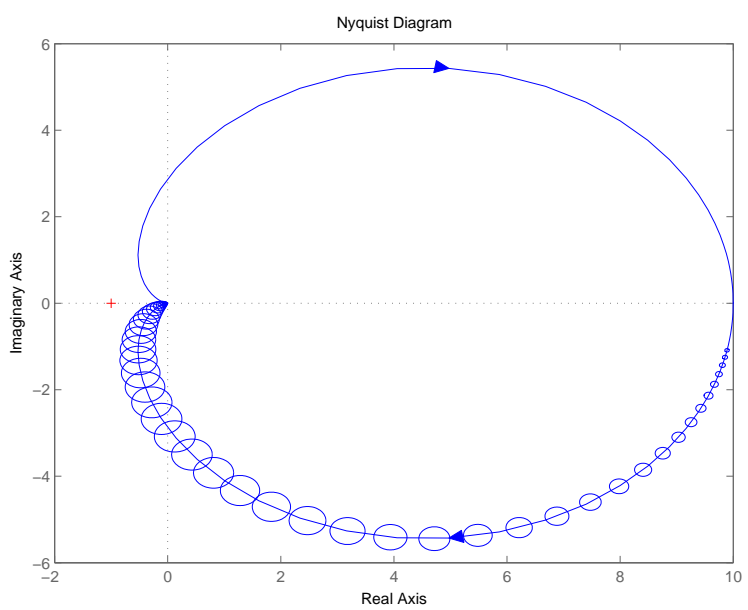

Fig. 15. Robust-stability chart showing loop transfer function and multiplicative uncertainty bound. As long as the uncertainty "smear" along the plot of $L(j \omega)$ does not include the $s=-1$ point, the system stability is robust to uncertainty.

A bound on the multiplicative uncertainty can be developed from the perturbed and nominal plants

$$
\left|\frac{L^{\prime}(j \omega)}{L(j \omega)}-1\right| \leq\left|W_{2}(j \omega)\right|
$$

For robust stability, the $s=-1$ point must lie outside the circles of radius $\left|W_{2}\left(j \omega_{k}\right) L\left(j \omega_{k}\right)\right|$ centered at the corresponding points $L\left(j \omega_{k}\right)$ for all frequencies $\omega_{k}$. In above example, the uncertainty of the unmodeled high-frequency pole can be bounded by

$$
\left|\frac{1}{\tau s+1}-1\right|=\left|\frac{\tau s}{\tau s+1}\right| \leq\left|\frac{\tau_{m} s}{\tau_{m} s+1}\right|
$$

As long as the uncertainty circles along of the plot of $L(j \omega)$ do not include the $s=-1$ point (as demonstrated in Figure 15), the system stability is robust to uncertainty. 


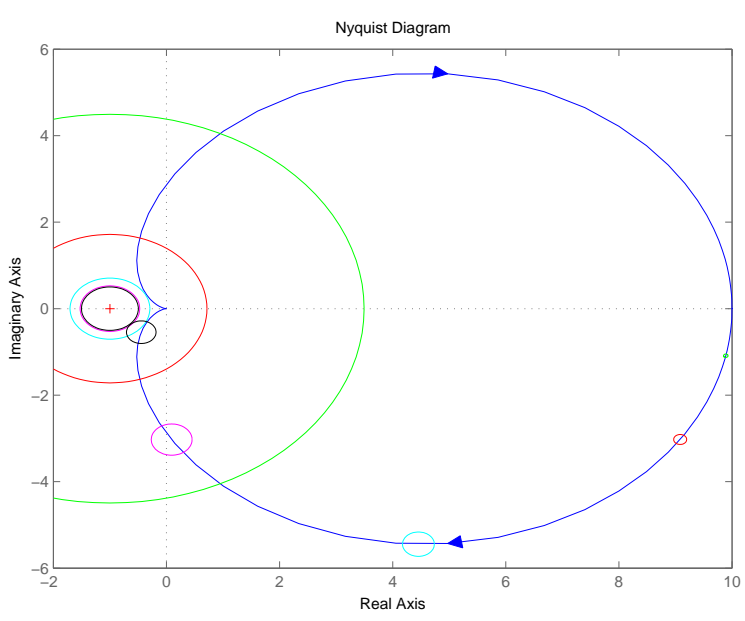

Fig. 16. Robust-performance chart. At each frequency, the robust-stability circle of radius $\left|W_{2} L\right|$ and centered at $L$ must be outside the performance circle of radius $\left|W_{1}\right|$ and centered at $s=-1$.

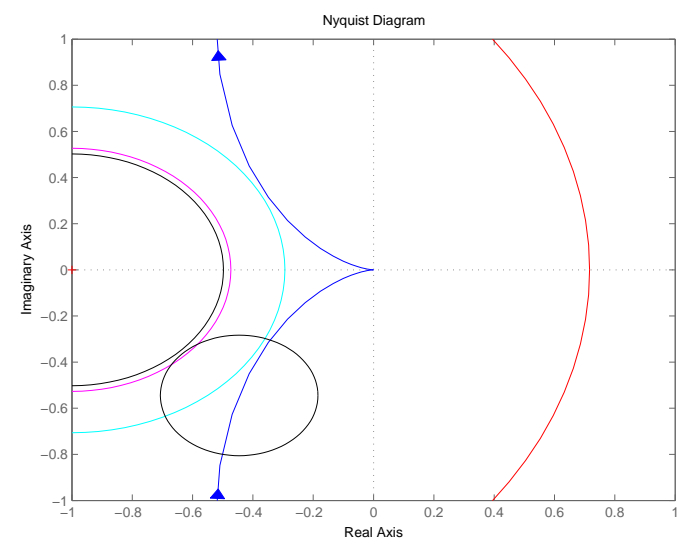

Fig. 17. Magnification of robust-performance chart in Figure 16. The black circles in this plot show the closest approach, but no intersection. These circles must be drawn and checked for all frequencies.

For robust performance the circles created by the sensitivity bound are included in the plot, as shown in Figure 16. The concept of a "smeared" frequency response (as shown in Figure 15) cannot be used.

Therefore, it is required that at each frequency $\omega_{k}$, the disc of uncertainty (with radius $\left.\left|L\left(j \omega_{k}\right) W_{2}\left(j \omega_{k}\right)\right|\right)$ around the loop transfer function $L\left(j \omega_{k}\right)$ must not intersect the circle of radius $\left|W_{1}\left(j \omega_{k}\right)\right|$ centered at $s=-1$. The robust-performance diagram in Figure 16 shows that there is no intersection, even at the closest approach. However, the circles at all frequencies must be examined.

Unless these circles are drawn and checked for all frequencies, robust performance cannot be guaranteed. A threedimensional model of the robust-performance chart (as shown in Figure 18) clears up the potential ambiguity. The bounds on the sensitivity function and multiplicative plant uncertainty become solids that must not intersect. The sensitivity bound becomes a cone centered on $s=-1$, and the multiplicative plant uncertainty is represented by the thickness of the loop transfer function "snake." The vertical axis corresponds to

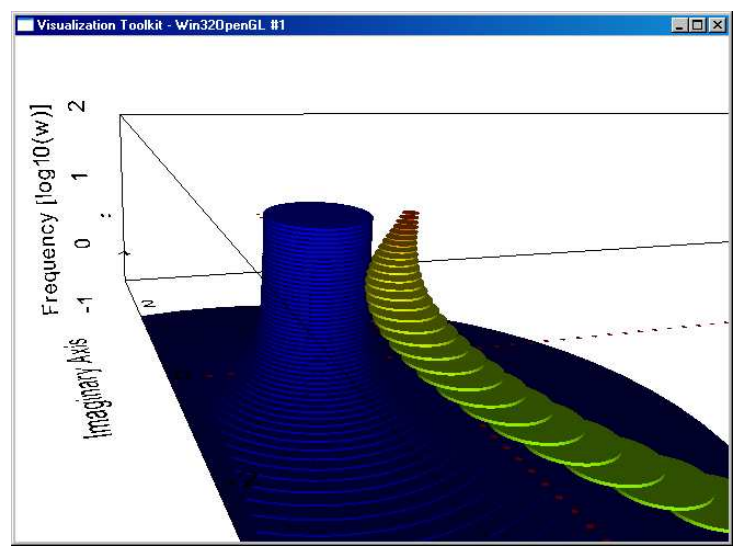

Fig. 18. Three-dimensional model of the robust-performance chart in Figure 16. Examining this model for intersections of the corresponding solids indicates the success or failure of the robust-performance design.

frequency, thus the bounds are displayed on the plot for all frequencies. Robust-performance is guaranteed if the uncertainty snake does not intersect the sensitivity cone. Using a three-dimensional model, the absence of this intersection can be readily examined.

\section{ACKNOWLEDGMENTS}

The authors' understanding of robust control has been greatly enhanced by many helpful conversations with Professor Leonard Gould.

Special thanks to Hubert James, Nathaniel Nichols, Ralph Phillips, and McGraw-Hill for putting their classic text [1] in the public domain ten years after the original publication, thus allowing us to freely reprint Figure 1.

The visualizations presented here were rendered using the Visualization Toolkit [4], a freely available open-source graphics package (http://www.vtk.org/).

\section{REFERENCES}

[1] Hubert M. James, Nathaniel B. Nichols, and Ralph S. Phillips. Theory of Servomechanisms, volume 25 of MIT Radiation Laboratory Series. McGraw-Hill, New York, 1947.

[2] Albert C. Hall. The Analysis and Synthesis of Linear Servomechanisms. Technology Press, M.I.T., Cambridge, MA, 1943.

[3] John C. Doyle, Bruce A. Francis, and Allen R. Tannenbaum. Feedback Control Theory. Macmillan, New York, 1992.

[4] W. J. Schroeder, K. M. Martin, and W. E. Lorensen. The design and implementation of an object-oriented toolkit for $3 \mathrm{~d}$ graphics and visualization. In IEEE Visualization, pages 93-100, 1996. 